\title{
Retrospective Analysis of Prognostic Factors in Adult Glioblastoma Multiforme: A Single Institution Experience
}

\author{
Mohamed S. Abd El Moumen 1, Lobna R. Ezz El Arab 2, Nesreen Mosalam 2, Ahmed \\ Gaballah $^{2}$
}

\author{
${ }^{1}$ Oncology Department, Galaa Military Hospital, Cairo, Egypt; ${ }^{2}$ Clinical Oncology Department, \\ Faculty of Medicine, Ain Shams University, Cairo, Egypt
}

Background: Primary brain tumors represent $2 \%$ of cancers in adults. Glioblastoma Multiforme (GBM) is the most frequent among these tumors. Different prognostic factors have been identified including age, performance status, extent of surgery and genetic factors.

Aim: To analyze treatment outcome and prognostic factors in adult patients with GBM treated at a single institution.

Methods: We retrospectively collected the data of patients treated for GBM form January 2012 till December 2016. During this 5-years period, 111 patients were identified and the data of 93(84\%) of them was complete and included in the analysis.

Results: Males represented $67 \%$ of patients, their median age was 52 years and the Eastern Cooperative Oncology Group (ECOG) performance status was 1, 2 and 3 in 48\%, 40\% and 12\% respectively. Only $4.3 \%$ of patients underwent complete surgical resection, $38 \%$ underwent partial resection and 58\% underwent biopsy. Post-operative treatment was radiotherapy alone in $30 \%$ of patients and chemo-radiotherapy in $70 \%$. The median progression-free survival (PFS) and overall survival (OS) were 8 months (95\% Confidence Interval: 6.678-9.322) and 10 months (95\% Confidence Interval: 7.522-12.487), respectively. Longer PFS was associated with age $<50$ years, better baseline ECOG performance status, partial / complete excision, no corticosteroids dependence, and post-operative chemo-radiotherapy $(\mathrm{p}=0.012,0.001,0.025,<0.001$ and 0.038; respectively). Similarly OS was better in association with age <50 years, better baseline ECOG performance status, partial / complete excision, no corticosteroid dependency and post-operative chemo-radiotherapy $(p=0.002,0.032,0.048$, $<0.001$ and 0.024 ; respectively).

Conclusion: Glioblastoma Multiforme remains an aggressive disease with high mortality rate and poor outcome. Complete resection and adjuvant chemo-radiotherapy improve PFS and OS.

Keywords: Glioblastoma Multiforme, Prognosis, Egypt

Corresponding author: Dr. Ahmed Gaballah; Clinical Oncology Department, Faculty of Medicine, Ain Shams University, Cairo, Egypt. Email: Ahmed_gaballah@med.asu.edu.eg

Submitted: 25-November-2018, Revised: 10-January-2019, Accepted: 13-January-2019, Published online: 2-February-2019

\section{INTRODUCTION}

Primary central nervous system (CNS) tumors account for $2 \%$ of all malignancies in adults. About $50 \%$ of them are gliomas. Glioblastoma multiforme (GBM) is derived from neuroepithelial cells and is the most frequent and deadly primary CNS malignancy in adults 1. It accounts for $60-70 \%$ of gliomas in the adult population ${ }^{2}$. In the United States, the age-adjusted GBM incidence rate is 3.97 cases per 100,000 for males and 2.53 cases per 100,000 for females; accounting for $75 \%$ of all anaplastic gliomas in adults and $20 \%$ of primary CNS tumors ${ }^{3}$.

Treating GBM is challenging for oncologists with a short median overall survival (OS) of only 4.2 months for patients treated with surgery alone ${ }^{4}$. The median survival following chemo-radiotherapy remains also poor (14.6 months) ${ }^{1}$.

Prognostic factors for adult GBM identified by Scott et al included: age, extent of surgery (biopsy vs. complete resection) and Karnofsky performance status ${ }^{5}$.
According to prognostic factors, adult GBM patients were classified into 4 subgroups with a median OS ranging from 2.3 months for group IV (biopsy only with poor Karnofsky performance status < 70) to 9.3 months for subgroup I (complete surgical excision and age < 75.5 years).

Maximal safe surgical resection with postoperative radiotherapy (RT) and adjuvant temozolomide or carmustine wafers after resection remains the standard of care for the treatment of adult GBM. Despite this, the survival remains poor with a median OS from 12 to 15 months and a 2-year and 3-year survival rates of 3.3\% and $1.2 \%$ respectively ${ }^{1}$.

Dexamethasone dependency during RT has been reported to be an independent poor predictor of survival in patients with high grade gliomas ${ }^{6}$. In one study, patients who were "steroid dependent" after craniotomy had a 1.9 relative death risk compared to those who were off steroids post-operatively ${ }^{7}$. This was also reported before from our institution by Abdel Karim et al. They found that the dependency on corticosteroids is 
associated with significantly shorter OS $(\mathrm{p}<0.001)$ and progression-free survival (PFS) $(\mathrm{p}=0.035)^{8}$.

In this study we aimed at evaluating the different prognostic factors in adult GBM patients treated at a single Egyptian University hospital-based oncology service.

\section{METHODS}

The current study is a retrospective analysis of adult GBM patients treated at the Clinical Oncology Department of Ain Shams University, Cairo, Egypt; in the period between January 2012 and December 2016.

The inclusion criteria included: radiological and pathological diagnosis of GBM, age >18 years and complete medical records.

The data collected included: age, gender, baseline Eastern Cooperative Oncology Group (ECOG) performance status, tumor characteristics, type of surgery, postoperative treatment and dependence on corticosteroids. Data was analyzed to determine possible prognostic factors.

During the specified time period, 111 patients were treated for GBM at our institution. The final analysis included 93 (84\%) patients. Eighteen patients were excluded due to incomplete data or loss to follow up after initial diagnosis.

Data was analyzed using the Statistical Package for the Social Sciences (SPSS) version 20 (SPSS Inc., Chicago. IL). Numerical data were presented as mean and standard deviation (SD) or median and range as appropriate. Progression-free survival was calculated from the date of surgery/biopsy to the date of disease progression or death and OS from the date of surgery/biopsy to the date of death. Survival analysis was done using Kaplan-Meier method and survival correlation was assessed using log-rank test. Differences were considered significant if the $\mathrm{p}$-value is $<0.05$.

\section{RESULTS}

The median age of patients was 52 years (range: 1880) with a male to female ratio of $2: 1$. Most of the patients $(88.2 \%)$ presented with a good performance status (ECOG 1-2). The mean radiological tumor size at diagnosis was $5.15 \mathrm{~cm}( \pm 1.37)$ and $60 \%$ of patients had a tumor size $>5 \mathrm{~cm}$.

A minority $(4 \%)$ of patients underwent complete resection and $>1 / 2$ of them underwent biopsy only. All patients received postoperative radiotherapy. The radiotherapy was computerized tomography (CT)-based 3-dimensional conformal. Seventy-two percent of patients received conventional fractionation radiotherapy (60 Grays over 6 weeks) and 28\% received hypofractionated radiotherapy (45 grays in 15 fractions over 3 weeks). Seventy percent of patients continued after radiotherapy on adjuvant temozolomide for 6 months and the remaining did not received adjuvant temozolamide due to financial problems with the Ministry of Health support program. Concomitant temozolamide with radiotherapy was not supported as well. Details of patients and tumor characteristics as well as treatment received are illustrated in table 1.

Table 1: Patients and tumor characteristics

\begin{tabular}{|c|c|c|}
\hline & No. & $\%$ \\
\hline \multicolumn{3}{|l|}{ Age } \\
\hline$<50$ years & 42 & 45 \\
\hline$\geq 50$ years & 51 & 55 \\
\hline \multicolumn{3}{|l|}{ Gender } \\
\hline Female & 31 & 33.3 \\
\hline Male & 62 & 66.7 \\
\hline \multicolumn{3}{|l|}{ ECOG Performance status } \\
\hline 1 & 45 & 48 \\
\hline 2 & 37 & 39.8 \\
\hline 3 & 11 & 12.2 \\
\hline \multicolumn{3}{|l|}{ Largest tumor diameter } \\
\hline$<5 \mathrm{~cm}$ & 37 & 39.8 \\
\hline$\geq 5 \mathrm{~cm}$ & 56 & 60.2 \\
\hline \multicolumn{3}{|l|}{ Tumor Site } \\
\hline Frontal & 18 & 19 \\
\hline Parietal & 17 & 18 \\
\hline Temporal & 15 & 16 \\
\hline Occipitoparietal & 15 & 16 \\
\hline Temproparietal & 13 & 14 \\
\hline Frontoparietal & 12 & 13 \\
\hline Brainstem & 3 & 4 \\
\hline \multicolumn{3}{|l|}{ Type of surgery } \\
\hline Biopsy only & 53 & 57 \\
\hline Partial resection & 36 & 38.7 \\
\hline Complete resection & 4 & 4.3 \\
\hline \multicolumn{3}{|l|}{ Steroidal dependency } \\
\hline No & 30 & 32 \\
\hline Yes & 63 & 68 \\
\hline \multicolumn{3}{|l|}{ Type of postoperative treatment } \\
\hline Radiotherapy alone & 28 & 30 \\
\hline Radiotherapy + temozolomide & 65 & 70 \\
\hline \multicolumn{3}{|l|}{ Radiotherapy fractionation type } \\
\hline Conventional & 67 & 72 \\
\hline Hypofractionation & 26 & 28 \\
\hline
\end{tabular}

The median PFS for all patients was 8 months (Standard Error [SE]: 0.675; 95\% CI: 6.678-9.322) and the median OS was 10 months (SE: $1.264 ; 95 \%$ CI: 7.522-12.487) (figures 1 and 2, respectively).

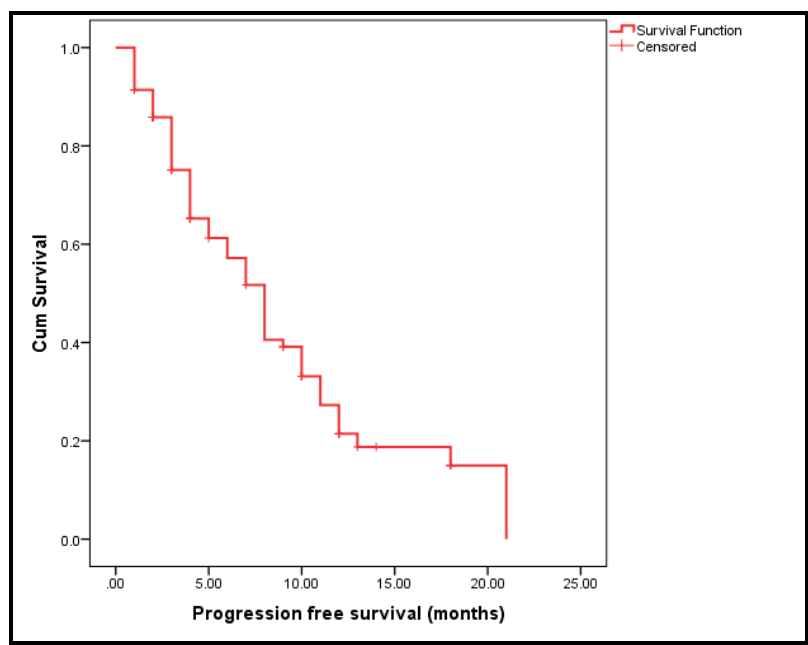

Figure 1: Kaplan-Meier progression-free survival curve for all patients 


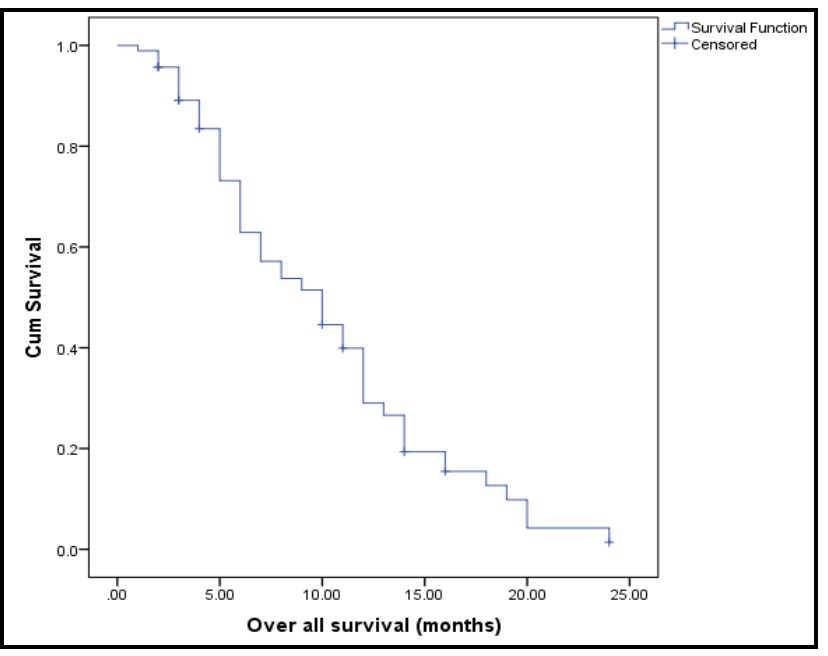

Figure 2: Kaplan-Meier overall survival curve for all patients

Tables 2 and 3 illustrate PFS and OS according to the studied variables. Progression-free survival differed significantly according to age, baseline ECOG performance status, type of surgery, adjuvant temozolomide therapy and steroidal dependency ( $\mathrm{p}=$ $0.012,0.001,0.025,0.038$ and $<0.001$; respectively) (figure 3). Overall survival differed significantly according to age, baseline ECOG performance status, type of surgery, steroidal dependency and adjuvant temozolomide therapy $(\mathrm{p}=0.002,0.032,0.048,<0.001$ and 0.024 ; respectively) (figure 4 ).
Progression-free survival and OS did not differ significantly according to gender, tumor size, site of tumor or type of radiotherapy fractionation.

\section{DISCUSSION}

In our study the median age of patients was 52 years which is younger than that reported by Caloglu et al ${ }^{9}$, but similar to another study from Iran. Most of the patients presented with good performance status (ECOG 1-2), similar to the results of Ahmadloo et al ${ }^{10}$. Eightysix percent of patients included in their study had and an ECOG performance status score from 0 to 2 .

The rate of gross total tumor resection among patients included in this study was only $4 \%$ which is much less than the $53 \%$ reported by Fekete et al ${ }^{11}$. This may be explained by the large mean tumor size at diagnosis in our population which was $5 \mathrm{~cm}$.

In our study, younger patients $(<50$ years) had better OS of 12 months and PFS of 9 months when compared to older patients who had OS of 7 months and PFS of 7 months. This is similar to the findings of $\mathrm{Li}$ et al ${ }^{12}$. This is likely due to the ability of younger patients to tolerate adjuvant chemotherapy and radiotherapy after complete or partial surgical resection of the tumor.

Better baseline ECOG performance status was associated with significantly better PFS and OS in our patients, which is in concordance with the results of the results of the study conducted by Ahmadloo et al ${ }^{10}$.

Table 2: Progression-free survival according to the studied variables

\begin{tabular}{|c|c|c|c|c|c|c|c|}
\hline & & \multirow[t]{3}{*}{ No. } & \multicolumn{4}{|c|}{ Progression free survival (months) } & \multirow[t]{3}{*}{ P value } \\
\hline & & & \multirow[t]{2}{*}{ Median } & \multirow[t]{2}{*}{ SE } & \multicolumn{2}{|c|}{$95 \% \mathrm{CI}$} & \\
\hline & & & & & Lower & Upper & \\
\hline \multirow[t]{2}{*}{ Age } & $<50$ yrs & 42 & 9 & 1.357 & 6.34 & 11.66 & 0.012 \\
\hline & $\geq 50 \mathrm{yrs}$ & 51 & 7 & 0.986 & 5.067 & 8.933 & \\
\hline \multirow[t]{2}{*}{ Gender } & Females & 31 & 8 & 1.473 & 5.112 & 10.888 & 0.355 \\
\hline & Males & 62 & 8 & 0.752 & 6.527 & 9.473 & \\
\hline \multirow[t]{3}{*}{ Baseline ECOG performance status } & 1 & 45 & 10 & 1.413 & 7.231 & 12.769 & 0.001 \\
\hline & 2 & 37 & 8 & 0.686 & 6.656 & 9.344 & \\
\hline & 3 & 11 & 4 & 0.621 & 2.783 & 5.217 & \\
\hline \multirow[t]{2}{*}{ Tumor size } & $<5 \mathrm{~cm}$ & 37 & 8 & 0.666 & 6.695 & 9.305 & 0.635 \\
\hline & $\geq 5 \mathrm{~cm}$ & 56 & 7 & 0.864 & 5.306 & 8.694 & \\
\hline \multirow[t]{7}{*}{ Tumor site } & Frontal & 18 & 13 & 2.926 & 4.266 & 15.734 & 0.3 \\
\hline & Parietal & 17 & 6 & 1.146 & 3.754 & 8.246 & \\
\hline & Temporal & 15 & 10 & 2.443 & 8.211 & 17.789 & \\
\hline & Occipitoparietal & 15 & 5 & 1.788 & 1.495 & 8.505 & \\
\hline & Temproparietal & 13 & 5 & 2.271 & 0.549 & 9.451 & \\
\hline & Frontoparietal & 12 & 7 & 1.923 & 3.231 & 10.769 & \\
\hline & Brainstem & 3 & 3 & 1.633 & 0 & 6.201 & \\
\hline \multirow[t]{2}{*}{ Type of surgery } & Biopsy & 53 & 11.45 & 1.275 & 8.954 & 13.952 & 0.025 \\
\hline & Partial /complete resection & 30 & 15.31 & 1.030 & 13.295 & 17.332 & \\
\hline \multirow[t]{2}{*}{ Steroidal dependency } & No & 30 & 13.91 & 1.164 & 11.631 & 16.194 & $<0.001$ \\
\hline & $\overline{\text { Yes }}$ & 63 & 5.58 & 0.608 & 4.386 & 6.770 & \\
\hline \multirow[t]{2}{*}{ Type of postoperative treatment } & Radiotherapy group & 28 & 9 & 0.72 & 7.588 & 10.412 & 0.038 \\
\hline & Radiotherapy + temozolomide & 65 & 14 & 1.714 & 10.64 & 17.36 & \\
\hline \multirow[t]{2}{*}{ Radiotherapy protocols } & Conventional & 67 & 5 & 2.271 & 0.549 & 9.451 & 0.214 \\
\hline & Hypofractionation & 26 & 7 & 1.923 & 3.231 & 10.769 & \\
\hline
\end{tabular}


Table 3: Overall survival according to the studied variables

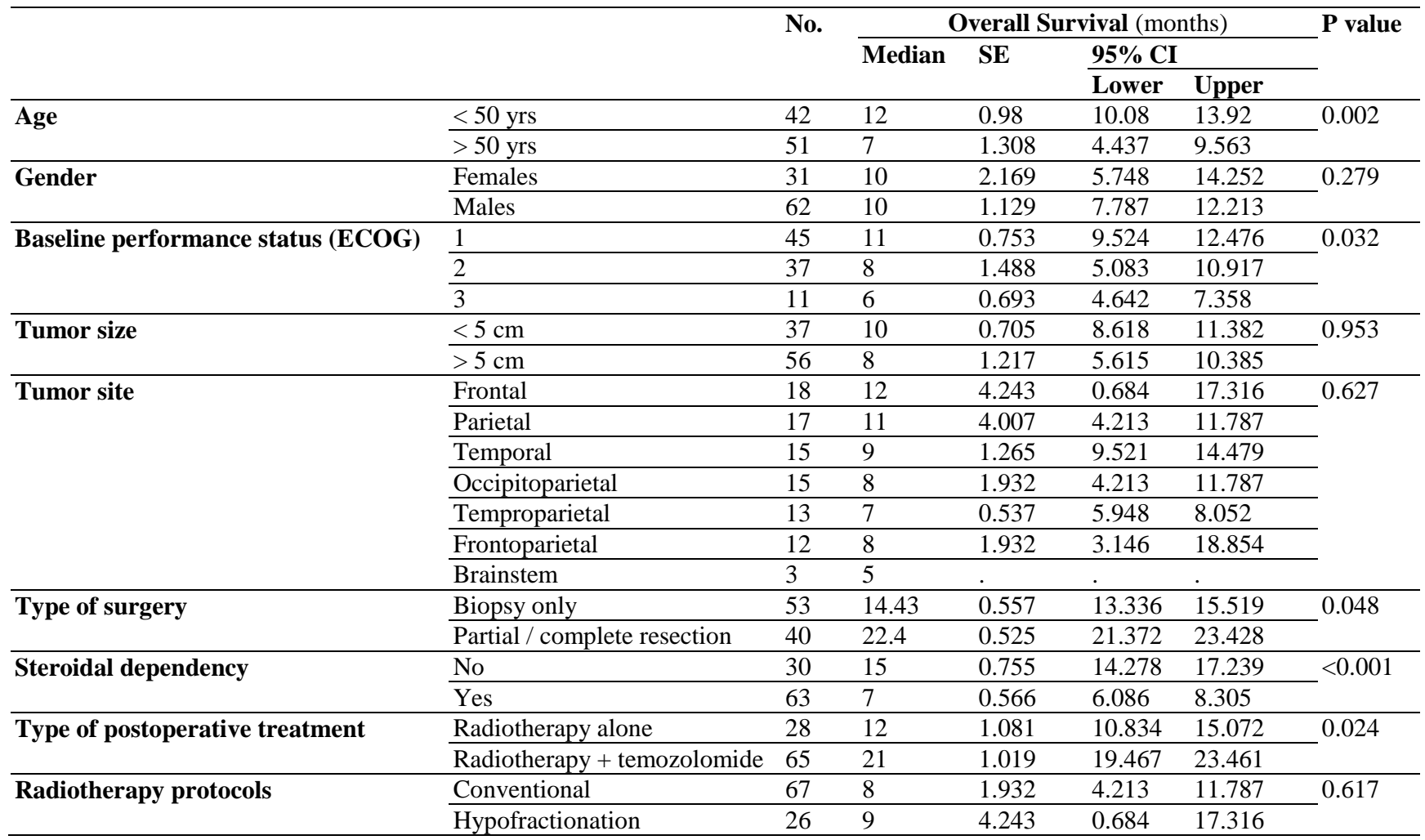
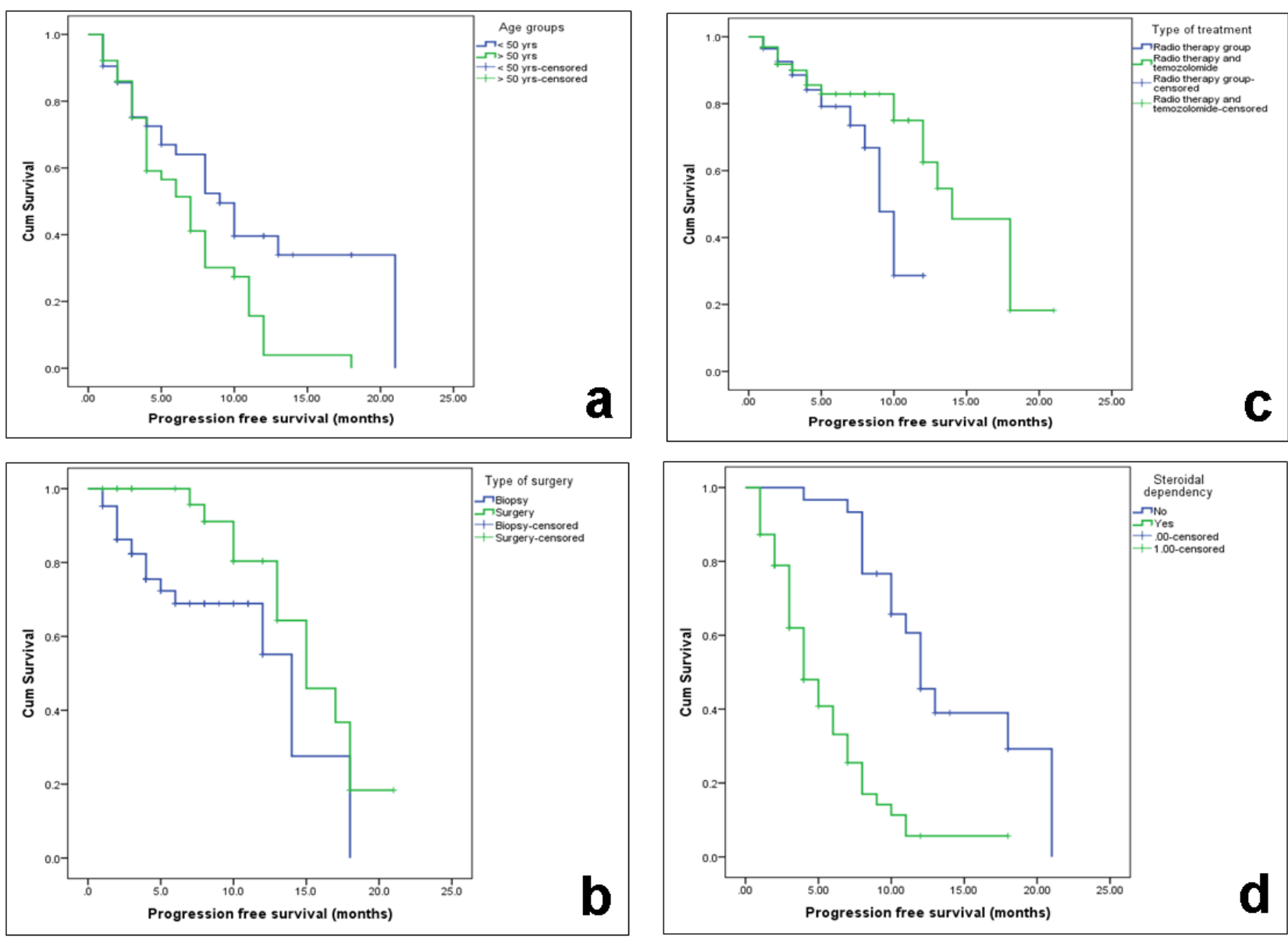

Figure 3: Kaplan-Meier progression free survival curves according to: a) age, b) type of surgery, c) type of postoperative treatment and d) steroidal dependency 

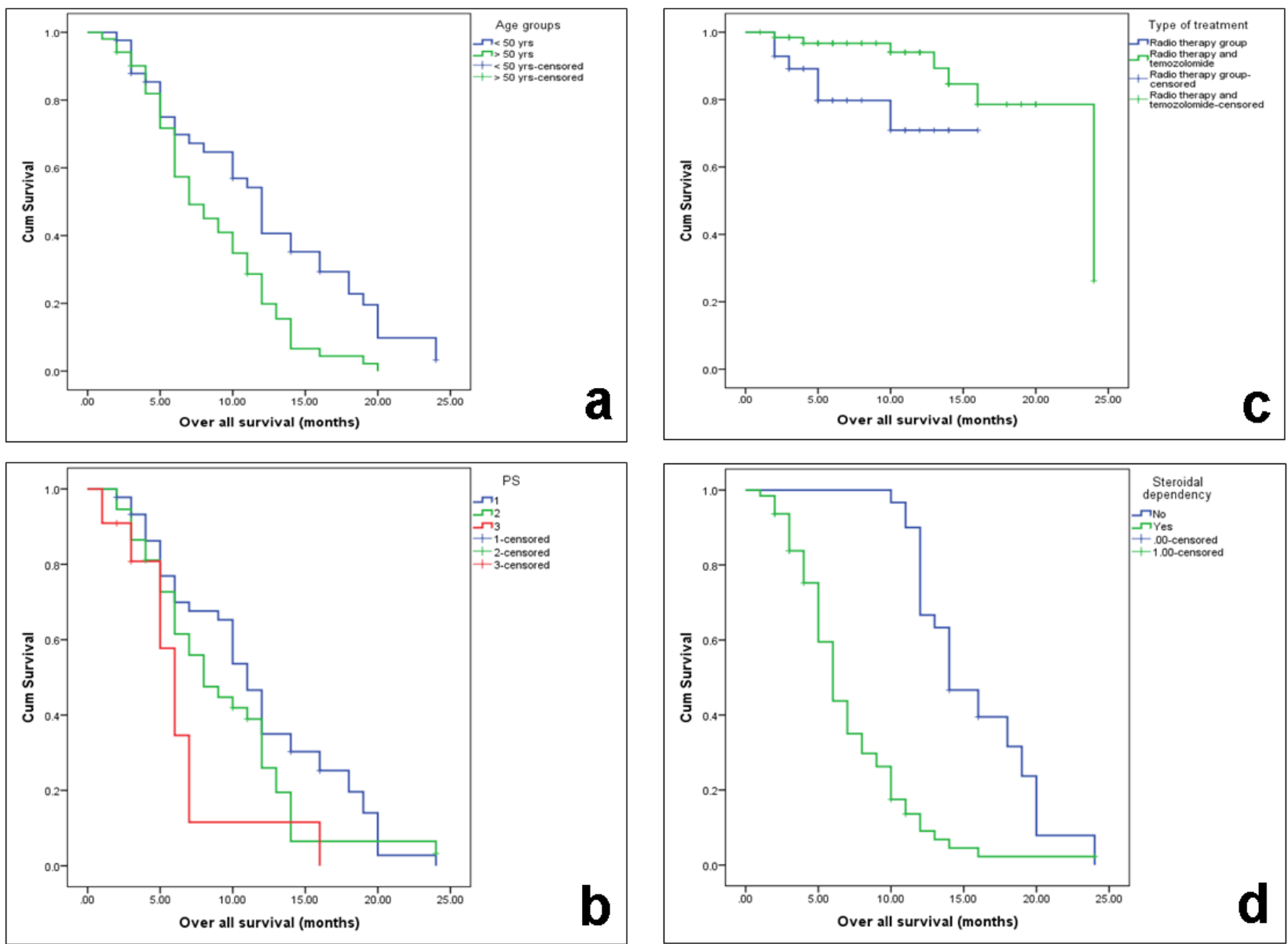

Figure 4: Kaplan-Meier progression-free survival curves according to: a) age, b) performance status, c) type of postoperative treatment and d) steroidal dependency

Surgical resection of malignant gliomas remains one of the most important prognostic factors. In a clinical trial, the absence of postoperative enhancing lesion by magnetic resonance imaging (MRI) significantly improved survival (median OS 17.9 vs. 12.9 months for residual disease by MRI, p < 0.001) ${ }^{13}$. This data is similar to our data where patients who underwent surgical excision had a median OS of 22 months compared to 14 months for those who underwent biopsy only.

For years, resection followed by postoperative radiotherapy was the standard for treating GBM. In late 1970s, trials began to evaluate the role of chemotherapy ${ }^{14}$. In the pivotal phase III European Organisation for Research and Treatment of Cancer / National Cancer Institute of Canada (EORTC-NCIC) study, the addition of temozolomide as concurrent and adjuvant treatment improved survival for GBM patients and this survival advantage was maintained after 5 years of follow up ${ }^{1}$. In our study, patients who received adjuvant temozolomide had significantly higher median OS of 21 months compared to 12 months for those who did not receive adjuvant chemotherapy.

Of note, in our study patients who received hypofractionation radiotherapy had median a OS of 8 months which is close to that of those who received conventional radiotherapy ( 9 months) and the difference was not statistically significant.

\section{Conclusion}

Glioblastooma multiforme remains an aggressive disease with low PFS and OS. Total resection and trimodality therapy provide the best approach to improve PFS and OS.

\section{Conflict of interest}

None to declare.

\section{REFERENCES}

1. Stupp R, Mason WP, van den Bent MJ, et al. Radiotherapy plus concomitant and adjuvant temozloamide for glioblastoma. N Engl J Med. 2005; 352(10): 987-996.

2. $\mathrm{Li} \mathrm{R}, \mathrm{Li} \mathrm{H}$, Yan $\mathrm{W}$, et al. Genetic and clinical characteristics of primary and secondary glioblastoma is associated with differential molecular subtype distribution. Oncotarget. 2015; 6(9): 7318-7324.

3. Furnari FB, Fenton T, Bachoo RM, et al. Malignant astrocytic glioma: genetics, biology, and paths to treatment. Genes Dev. 2007; 21(21): 2683-2710. 
4. Arvold ND, Reardon DA. Treatment options and outcomes for glioblastoma in the elderly patient. Clin Interv Aging. 2014; 9: 357-367.

5. Scott JG, Bauchet L, Fraum TJ, et al. Recursive partitioning analysis of prognostic factors for glioblastoma patients aged 70 years or older. Cancer. 2012; 118(22): 5595-5600

6. Hohwieler SM, Freidberg SR, Heatley GJ, Lo TC. Glucocorticoid dependency as a prognostic factor in radiotherapy for cerebral gliomas. Acta Oncol. 1989; 28(1): 51-55.

7. Watne K, Hannisdal E, Nome O, Hager B, Hirschberg H. Prognostic factors in malignant gliomas with special reference to intra-arterial chemotherapy. Acta Oncol. 1993; 32(3): 307-310.

8. Abdel Karim K, Kelany MR, Abdelrahman O, El-Adawy AM. Corticosteroids administration as a prognostic factor in glioblastoma patients: An Egyptian experience. Ann Oncol. 2018; 29 (suppl 8): viii126-viii127.

9. Caloglu M, Yurut-Caloglu V, Karagol H, Bayir-Angin G, Turan FN, Uzal C. Prognostic factors other than the performance status and age for glioblastoma multiforme: a single institution experience. J BUON. 2009; 14(2): 211218.

10. Ahmadloo N, Kani AA, Mohammadianpanah M, et al. Treatment outcome and prognostic factors of adult glioblastoma multiforme. J Egypt Natl Canc Inst. 2013; 25(1): 21-30.

11. Fekete B, Werlenius K, Orndal C, Rydenhag B. Prognostic factors for glioblastoma patients - a clinical population-based study. Acta Neurol Scand. 2016; 133(6) : 434-441

12. Li SW, Qiu XG, Chen BS, et al. Prognostic factors influencing clinical outcomes of glioblastoma multiforme. Chin Med J (Engl). 2009; 122(11): 1245-1249.

13. Stummer W. Mechanisms of tumor-related brain edema. Neurosurg Focus. 2007; 22(5): E8.

14. Laperriere N, Zuraw L, Cairncross G, et al. Radiotherapy for newly diagnosed malignant glioma in adults: a systematic review. Radiother Oncol. 2002; 64(3): 259273. 